\title{
MULTIVARIATE COMPOUND POISSON DISTRIBUTIONS AND INFINITE DIVISIBILITY
}

\author{
BY \\ BJøRN SUNDT \\ Vital Forsikring $A S A$
}

\begin{abstract}
In this note we give a multivariate extension of the proof of Ospina \& Gerber (1987) of the result of Feller (1968) that a univariate distribution on the non-negative integers is infinitely divisible if and only if it can be expressed as a compound Poisson distribution.
\end{abstract}

\section{INTRODUCTION}

Feller (1968) showed that a univariate distribution on the non-negative integers is infinitely divisible if and only if it can be expressed as a compound Poisson distribution. Ospina \& Gerber (1987) gave a new proof based on the recursions of Panjer (1980) for compound Poisson distributions and De Pril (1985) for $n$-fold convolutions. In the present paper we shall extend the proof of Ospina \& Gerber (1987) to multivariate distributions, using Sundt's (1999) multivariate extension of the recursions of Panjer and De Pril.

Before turning to infinite divisibility in Section 3 we present the recursions of Sundt (1999) in Section 2.

In this note we shall represent discrete probability distributions by their probability functions. For convenience we shall usually mean the probability function when referring to a distribution.

\section{Multivariate RECURSIONS}

In this note a vector will be denoted by a bold-face letter and the sum of its elements by that letter with a tilde, e.g. $\tilde{\mathbf{x}}$ is the sum of the elements of the vector $\mathbf{x}$. We let $\mathbb{N}_{m}$ denote the set of all $m \times 1$ vectors where all elements are non-negative integers, and introduce $\mathbb{N}_{m+}=\mathbb{N}_{m} \sim\{\mathbf{0}\}$ with $\mathbf{0}$ denoting the $m \times 1$ vector with all elements equal to zero. For $j=1, \ldots, m$ we define $\mathbf{e}_{j}$ as the $m \times 1$ vector whose $j$ th element is one and all other elements are zero. By $\mathbf{y} \leq \mathbf{x}$ we shall mean that $\mathbf{x}-\mathbf{y} \in \mathbb{N}_{m}$, and by $\mathbf{y}<\mathbf{x}$ that $\mathbf{x}-\mathbf{y} \in \mathbb{N}_{m+}$. When we indicate the range for a vector, it is tacitly assumed that it is an element of $\mathbb{N}_{m}$. 
Let $f$ be a distribution on $\mathbb{N}_{m}$ with $f(0)>0$. Sundt (1999) showed that the $n$-fold convolution $f^{n *}$ can be evaluated by the recursion

$$
\begin{gathered}
f^{n *}(\mathbf{x})=\frac{1}{f(\mathbf{0})} \sum_{\mathbf{0}<\mathbf{y} \leq \mathbf{x}}\left((n+1) \frac{\tilde{\mathbf{y}}}{\tilde{\mathbf{x}}}-1\right) f(\mathbf{y}) f^{n *}(\mathbf{x}-\mathbf{y}) \quad\left(\mathbf{x} \in \mathbb{N}_{m+}\right) \\
f^{n *}(\mathbf{0})=f(\mathbf{0})^{n} .
\end{gathered}
$$

In the univariate case $(m=1)$ this recursion was presented by De Pril (1985).

The compound Poisson distribution with Poisson parameter $\lambda>0$ and severity distribution $h$ on $\mathbb{N}_{m+}$ is the distribution $g$ on $\mathbb{N}_{m}$ given by

$$
g(\mathbf{x})=\sum_{n=0}^{\tilde{\mathbf{x}}} \frac{\lambda^{n}}{n !} e^{-\lambda} h^{n *}(\mathbf{x}) ; \quad\left(\mathbf{x} \in \mathbb{N}_{m}\right)
$$

in particular we have

$$
g(\mathbf{0})=e^{-\lambda} .
$$

From formula (3.8) in Sundt (1999) we obtain

$$
g(\mathbf{x})=\frac{\lambda}{\tilde{\mathbf{x}}} \sum_{\mathbf{0}<\mathbf{y} \leq \mathbf{x}} \tilde{\mathbf{y}} h(\mathbf{y}) g(\mathbf{x}-\mathbf{y}), \quad\left(\mathbf{x} \in \mathbb{N}_{m+}\right)
$$

which together with (2) can be applied for recursive evaluation of $g$. In the univariate case this recursion was described by Panjer (1980).

\section{INFINITE DIVISIBILITY}

A distribution $g$ on $\mathbb{N}_{m}$ is said to be infinitely divisible if there for each positive integer $n$ exists a distribution $g_{n}$ on $\mathbb{N}_{m}$ such that $g=g_{n}^{n *}$.

Lemma 1. If a distribution on $\mathbb{N}_{m}$ is infinitely divisible, then it has a positive probability at zero.

Proof. If $g$ is an infinitely divisible distribution on $\mathbb{N}_{m}$, then for each positive integer $n$ there exists a distribution $g_{n}$ on $\mathbb{N}_{m}$ such that $g=g_{n}^{n *}$. In particular we have $g(\mathbf{0})=g_{n}(\mathbf{0})^{n}$, that is,

$$
g_{n}(\mathbf{0})=g(\mathbf{0})^{1 / n}
$$

Assume that $g(\mathbf{0})=0$. Then $g_{n}(\mathbf{0})=0$ for all positive integers $n$. This implies that $g(\mathbf{x})=g_{n}^{n *}(\mathbf{x})=0$ when $\tilde{\mathbf{x}}<n$. As this should hold for all $n$, we must have $g(\mathbf{x})=0$ for all $\mathbf{x} \in \mathbb{N}_{m}$, and then $g$ cannot be a distribution. Hence we must have $g(\mathbf{0})>0$, which proves the lemma.

Q.E.D. 
The following theorem follows from Theorem 2.1 in Horn \& Steutel (1978). In the univariate case it has been proved by Feller (1968) and Ospina \& Gerber (1987). Our proof is a generalisation of the proof of Ospina \& Gerber.

Theorem 1. A non-degenerate distribution on $\mathbb{N}_{m}$ is infinitely divisible if and only if it can be expressed as a compound Poisson distribution with severity distribution on $\mathbb{N}_{m+}$.

Proof. For each positive integer $n$, a compound Poisson distribution with parameter $\lambda>\mathbf{0}$ and severity distribution $h$ on $\mathbb{N}_{m+}$ is the $n$-fold convolution of a compound Poisson distribution with parameter $\lambda / n$ and severity distribution $h$. Hence a compound Poisson distribution with severity distribution on $\mathbb{N}_{m+}$ is always infinitely divisible.

We now assume that $g$ is a non-degenerate, infinitely divisible distribution on $\mathbb{N}_{m}$. Then for each positive integer $n$ there exists a distribution $g_{n}$ on $\mathbb{N}_{m}$ such that $g=g_{n}^{n *}$. Lemma 1 gives that $g(\mathbf{0})>0$. From (1) and (4) we obtain

$$
g(\mathbf{x})=\frac{1}{g(\mathbf{0})^{1 / n}} \sum_{\mathbf{0}<\mathbf{y} \leq \mathbf{x}}\left((n+1) \frac{\tilde{\mathbf{y}}}{\tilde{\mathbf{x}}}-1\right) g_{n}(\mathbf{y}) g(\mathbf{x}-\mathbf{y}) . \quad\left(\mathbf{x} \in \mathbb{N}_{m+}\right)
$$

Introduction of $h_{n}=n g(\mathbf{0})^{-1 / n} g_{n} / \lambda$ with $\lambda=-\ln g(\mathbf{0})$ gives

$$
g(\mathbf{x})=\lambda \sum_{0<\mathbf{y} \leq \mathbf{x}}\left(\left(1+\frac{1}{n}\right) \frac{\tilde{\mathbf{y}}}{\tilde{\mathbf{x}}}-\frac{1}{n}\right) h_{n}(\mathbf{y}) g(\mathbf{x}-\mathbf{y}), \quad\left(\mathbf{x} \in \mathbb{N}_{m+}\right)
$$

from which we obtain

$$
h_{n}(\mathbf{x})=\frac{1}{g(\mathbf{0})}\left(\frac{g(\mathbf{x})}{\lambda}+\sum_{\mathbf{0}<\mathbf{y}<\mathbf{x}}\left(\frac{1}{n}-\left(1+\frac{1}{n}\right) \frac{\tilde{\mathbf{y}}}{\tilde{\mathbf{x}}}\right) h_{n}(\mathbf{y}) g(\mathbf{x}-\mathbf{y})\right) . \quad\left(\mathbf{x} \in \mathbb{N}_{m+}\right)
$$

In particular, for $j=1, \ldots, m$ we see that $h_{n}\left(\mathbf{e}_{j}\right)=\frac{g\left(\mathbf{e}_{j}\right)}{g(\mathbf{0}) \lambda}$ independent of $n$, and it follows by induction on $\mathbf{x}$ that $h(\mathbf{x})=\lim _{n \dagger \infty} h_{n}(\mathbf{x})$ exists and is finite for all $\mathbf{x} \in \mathbb{N}_{m+}$. By letting $n$ go to infinity in (5) we then obtain

$$
g(\mathbf{x})=\frac{\lambda}{\tilde{\mathbf{x}}} \sum_{\mathbf{0}<\mathbf{y} \leq \mathbf{x}} \tilde{\mathbf{y}} h(\mathbf{y}) g(\mathbf{x}-\mathbf{y}) . \quad\left(\mathbf{x} \in \mathbb{N}_{m+}\right)
$$

Hence, $g$ satisfies (3), and by the definition of $\lambda$ it also satisfies (2). We see that $\lambda>0$ and $h$ is a non-negative function on $\mathbb{N}_{m+}$. From (3) we obtain

$$
g(\mathbf{x}) \geq \lambda g(\mathbf{0}) h(\mathbf{x}), \quad\left(\mathbf{x} \in \mathbb{N}_{m+}\right)
$$


which gives

$$
c=\sum_{\mathbf{x} \in \mathbb{N}_{m+}} h(\mathbf{x}) \leq \sum_{\mathbf{x} \in \mathbb{N}_{m+}} \frac{g(\mathbf{x})}{\lambda g(\mathbf{0})}=\frac{1-g(\mathbf{0})}{\lambda g(\mathbf{0})}<\infty .
$$

As $g$ is non-degenerate, (3) gives that $h(\mathbf{x})>0$ for at least one $\mathbf{x} \in \mathbb{N}_{m+}$, so that $c>0$. Let $\bar{g}$ be the compound Poisson distribution with Poisson parameter $\lambda$ and severity distribution $\bar{h}=h / c$ on $\mathbb{N}_{m+}$. Then $\bar{g}$ sums to one. If $c<1$, then (3) gives that $g(\mathbf{x}) \leq \bar{g}(\mathbf{x})$ for all $\mathbf{x} \in \mathbb{N}_{m}$ with strict inequality for at least one $\mathbf{x}$, and then $g$ cannot sum to one, which is a contradiction. Analogously, we cannot have $c>1$. Therefore, $c=1, h=\bar{h}$, and $g=\bar{g}$. This proves that a nondegenerate, infinitely divisible distribution on $\mathbb{N}_{m}$ can always be expressed as a compound Poisson distribution with severity distribution on $\mathbb{N}_{m+}$.

This completes the proof of Theorem 1 .

Q.E.D.

The restriction to non-degenerate distributions in Theorem 1 can be dropped if we consider a distribution concentrated in zero as a compound Poisson distribution with Poisson parameter zero and any severity distribution on $\mathbb{N}_{m+}$.

Corollary 1. The elements of a random vector with infinitely divisible distribution on $\mathbb{N}_{m}$ are non-negatively correlated.

Proof. It is easily shown (cf. e.g. subsection 2C in Sundt (1999)) that the elements of a random vector with compound Poisson distribution with severity distribution on $\mathbb{N}_{m+}$ are non-negatively correlated, and then the corollary follows from Theorem 1 .

Q.E.D.

\section{REFERENCES}

DE PRIL, N. (1985) Recursions for convolutions of arithmetic distributions. ASTIN Bulletin 15, $135-139$.

FELLER, W. (1968) An introduction to probability theory and its applications. Vol. 1 (3. ed.) Wiley, New York.

Horn, R.A. \& Steutel, F.W. (1978) On multivariate infinitely divisible distributions. Stochastic Processes and their Applications 6, 139-151.

OSPINA, A.V. \& Gerber, H.U. (1987) A simple proof of Feller's characterization of the compound Poisson distributions. Insurance: Mathematics and Economics 6, 63-64.

PANJER, H. H. (1980) The aggregate claims distribution and stop-loss reinsurance. Transactions of the Society of Actuaries 32, 523-535.

SundT, B. (1999) On multivariate Panjer recursions. ASTIN Bulletin 29, 29-45.

\section{BJøRn SUNDT}

Vital Forsikring $A S A$

P.O. Box 250

N-1326 Lysaker

Norway 